\title{
SALUD
}

\section{Predictores de mortalidad en niños de 15 años o menos que adquirieron el VIH por transmisión materno-infantil en el Paraguay}

\author{
Gloria Aguilar ${ }^{1}$, Angélica Espinosa Miranda', George W. Rutherford', \\ Sergio Muñoz¹, Tania Samudio', Fernando Galeano', Anibal \\ Kawabata1', Carlos Miguel Ríos González
}

\section{Resumen}

Introducción: La pandemia de la infección por el virus de la inmunodeficiencia humana (VIH) se presenta actualmente como uno de los problemas más impactantes de salud pública a nivel mundial, con múltiples repercusiones que rebasan el ámbito sanitario. En el año 2015, 2,1 millones [1,8 millones-2,4 millones] de personas contrajeron la infección por el VIH en todo el mundo. Las nuevas infecciones en niños han descendido en un 50\% desde el 2010. A nivel mundial, 150000 [110 000-190 000] niños contrajeron la infección por el VIH en 2015, una cifra inferior a la de 2010, con 290000 [250 000-350 000] nuevos casos. En América Latina, a pesar de los avances en el control de la epidemia del VIH, la tendencia de la mortalidad de los niños con VIH y los predictores de la misma no han sido bien caracterizadas en los escasos estudios publicados hasta la fecha, en Brasil, en una cohorte de 15 años de seguimiento en Belo Horizonte, y otra realizada en Vitoria, mostraron una mortalidad global al finalizar el seguimiento que variaba de 9.7 y $15 \%$ respectivamente, mientras que Bologna R. y col en Argentina encontraron una tasa de mortalidad de 0.86 cada cien pacientes año y una tasa global de mortalidad del 3,4\%. Aunque los datos son bastante limitados para los países latinoamericanos, hay aún menos datos sobre la mortalidad de niños con infección por VIH transmitida perinatalmente en Paraguay. Hasta la fecha, los datos que se recopilan sobre los niños no han proporcionado una imagen clara de las tendencias en la mortalidad y los predictores de mortalidad para permitir a las autoridades sanitarias y del Programa Nacional de control de VIH/sida/ITS evaluar e implementar estrategias para reducir la mortalidad en niños que viven con el VIH.

Objetivo: Estimar la mortalidad y los predictores de mortalidad en los niños que adquirieron el VIH por transmisión materno-infantil en el Paraguay.

Material y Métodos: Se realizó un estudio observacional, de tipo cohorte retrospectiva, con seguimiento de personas viviendo con el VIH que tenían $<15$ años de edad al momento del reclutamiento entre los años 2000-2014. La población de estudio estuvo conformada por todos los niños menores

1. Dirección General de Investigación, Universidad Nacional de Caaguazú, Paraguay.

E-mail:dra.gloria.aguilar@gmail.com

DOI: 10.26885/rcei.foro.2017.161 
de 15 años de edad que adquirieron el VIH por transmisión madre a hijo diagnosticados de acuerdo a los criterios del Programa Nacional de Control de $\mathrm{VIH} / \mathrm{sida} / \mathrm{ITS}$ (nivel plasmático de RNA VIH-1 $>1000$ copias/ml en niños $<18$ meses y el diagnóstico confirmatorio con la detección de anticuerpos, 2 test de Elisa y un test confirmatorio (Western Blot o inmunofluorescencia en niños $\geq 18$ meses de edad) quienes recibieron atención en los Servicios de Atención Integral (SAI) para personas con VIH: Instituto de Medicina Tropical, Hospital Nacional de Itaugua, Hospital Regional de Ciudad del Este y Hospital Regional de Itapuá. Se obtuvieron los datos de los registros de historias clínicas. Los datos de óbitos se registraron de las historias clínicas y las confirmaciones de las muertes por los certificados de defunción. El outcome principal considerado fue la mortalidad, medida desde la fecha de nacimiento hasta la muerte, para esto fueron utilizadas las técnicas de sobrevida. Además se estimaron la incidencia de muerte al año, a los 5 años y a los 10 años del diagnóstico. Se utilizó el modelo de Cox para el análisis multivariado de los predictores de mortalidad usando las siguientes co-variables: edad al diagnóstico, residencia, periodo de diagnóstico, sexo, residencia, carga viral basal, estadio de infección, hemoglobina basal y edad al inicio de ARV, en meses

Resultados: Un total de 302 sujetos fueron incluidos, 216(71,5\%) de los sujetos se encontraban entre 0 y 5 años edad, $129(42,7 \%)$ se diagnosticaron en el 2010 o posterior y 131(43,3\%) entre el 2005 al 2009, 148(51,0\%)fueron del sexo masculino y $214(70,8 \%)$ residían en el área metropolitana. Desde el punto de vista virológico, $200(66,2 \%)$ presentaron una carga viral igual o mayor a 100.000 copias por $\mathrm{ml}$ y $241(79,8 \%)$ tuvieron niveles de hemoglobina mayor a $9 \mathrm{gr} / \mathrm{dl}$. De los 206 (82,2\%) que iniciaron ARV durante el periodo de estudio, $69(27,7 \%)$ fueron menores de 18 años en el momento de admisión.

El Tiempo total de seguimiento de los 302 niños fue de 2.522,5 años-persona. Se observaron 52 muertes, la tasa de mortalidad general fue de 2,06/ 100 años persona. Al 1er. Año se registraron 10 óbitos (3.3\% IC95\% 1,5-6,0), a los 5 años 32 óbitos (10.6\%IC95\% 7,3-14,6), a los 10 años 44 óbitos (14,50\% IC95\% 10,7-19,6). La mortalidad global al final del periodo fue de 17,2\%(IC: 13, 1\%21,9\%). 25 niños con VIH (8,8\%) fueron perdidos de seguimiento. En el modelo de riesgos proporcionales (Cox) aquellos niños con niveles de hemoglobina $<9 \mathrm{~g} / \mathrm{dl}$ en la línea de base tuvieron un riesgo de muerte dos veces mayor en comparación con aquellos con niveles $>9 \mathrm{~g} / \mathrm{dl}$ (HR: 2,27; intervalo de confianza $95 \%, 1.01-5.10)$. El riesgo de muerte fue $74 \%$ menor en los niños diagnosticados igual o posterior al año 2010 que los diagnosticados antes del año 2010. [HR = 0.26 (IC 95\%, 0.07-0-88)].

Conclusiones: La mortalidad de los niños con VIH en el Paraguay fue alta. El factor predictor de la mortalidad fue la hemoglobina $<9 \mathrm{~g} / \mathrm{dl}$ al momento del diagnóstico. Se deben fortalecer las estrategias para diagnosticar y tratar la infección por VIH en mujeres embarazadas en el Paraguay para prevenir la transmisión perinatal, mejorar el acceso al diagnóstico precoz de niños 
nacidos de infectados por VIH a través de la transmisión materno-infantil y el tratamiento de los que se han infectado antes de que desarrollen una enfermedad más avanzada. Además, fortalecer el soporte nutricional en los Servicios de atención integral del VIH y puede coadyuvar para la disminución de las muertes relacionadas al $\mathrm{VIH}$.

Palabras clave: mortalidad, niño, infecciones por VIH.

\section{Referencias}

Cotton, M. F., Violari, A, Otwombe, K. (2013). For the CHER study team. early time-limited antirretroviral therapy versus deferred therapy in South African infants infected with HIV: results from the children with HIV early antirretroviral (CHER) randomised trial. Lancet, (382), 1555-63.

Dunn, D., Woodburn, P. (2008). HIV pediatric prognostic markers collaborative study and the cascade collaboration. current $\mathrm{cd} 4$ and the short - term risk of AIDS and death before the availability of effective antiretroviral therapy in HIV-infected children and adults. J Infect Dis., 197(3), 398-404.

Moreira-Silva, S.F., Zandonade, E. \& Miranda, A. E. (2015). Mortality in children and adolescents vertically infected by HIV receiving care at a referral hospital in Vitoria, Brazil. BMC infectious diseases, 15(1), 155.

UNAIDS. (2017). Hoja informative 2016. Programme on HIV/AIDS. Recuperado de http://www.unaids.org/es/resources/fact-sheet

Violari, A., Cotton, M. F., Gibb, D. M., Babiker, A. G., Steyn, J., Madhi, S.A. (2008). Early antiretroviral therapy and mortality among HIV-infected infants. New England Journal of Medicine, 359(21), 2233-2244. 